www.jmscr.igmpublication.org

Impact Factor 5.84

Index Copernicus Value: 83.27

ISSN (e)-2347-176x ISSN (p) 2455-0450

crossref DOI: _https://dx.doi.org/10.18535/jmscr/v5i3.129

Journal Of Medical Science And Clinical Research

\title{
Early Prediction of Acute Kidney Injury by Clinical and Biochemical Parameters in Snakebite Patients
}

\author{
Authors \\ Dr Vinay S. Panchalwar ${ }^{*}$, Dr Chandrashekhar M. Atkar ${ }^{1}$, Dr Manu Vincent ${ }^{2}$ \\ ${ }^{1}$ Associate Professor, ${ }^{2}$ Junior Resident \\ Department of General Medicine, Government Medical College, Nagpur, Maharashtra, India- 440001 \\ Corresponding Author \\ Dr Vinay S. Panchalwar \\ Associate Professor, Department of General Medicine, Government Medical College, Nagpur, \\ Maharashtra, India-440001 \\ Email:vriankalwar@yahoo.com
}

\begin{abstract}
Aims and Objectives: The present study was undertaken to determine early clinical and biochemical predictors for Acute Kidney Injury (AKI) among snake bite patients at the time of hospital admission and to determine incidence of AKI in snake bite patients.

Methods: We analyzed 200 cases with a poisonous snake bite. Patients were classified into two groups according to the presence and absence of AKI (AKI and No-AKI). History, findings of clinical examination and investigations of all patients were recorded and results were statistically compared.

Results: Out of 200 patients of snake bite, 63 developed AKI (31.5\%). Among the clinical features, there was an independent positive association of AKI with bleeding tendencies, hypotension, wound complications and prolonged bite-to-initial Anti-Snake Venom (ASV) administration time. Similarly, among biochemical parameters, strong association of AKI was observed with hemoglobinuria, albuminuria, Red Blood Cells $(R B C)$ in urine, elevated International Normalized Ratio (INR), elevated indirect bilirubin, decreased platelets and prolonged 20 min Whole Blood Clotting Time (WBCT) test.

Conclusions: Clinical manifestations (bleeding manifestations, hypotension, tenderness, progressive swelling and wound complications) and biochemical parameters (hemoglobinuria, albuminuria, RBC in urine, elevated INR, elevated indirect bilirubin, decreased platelets and prolonged 20min WBCT test) can be considered as early predictors of AKI among snake bite patients including delayed presentation to hospital resulting in prolonged bite-to-initial ASV administration time.

Keywords: Acute Kidney Injury (AKI), Clinical and biochemical predictors, Snake bite, Anti-Snake venom $(A S V)$.
\end{abstract}

\section{Introduction}

Snake bite induced morbidity and mortality is a common health care problem all over the world especially in tropical and sub-tropical areas. The most conservative country specific estimates put India on top with maximum number of snake bite related envenomations and deaths in a year. About $19.2 \%$ and $55 \%$ of all snakebite related envenomations and deaths, respectively, that occur annually all over the world take place in India ${ }^{(1)}$. A recently published survey of snake bite related mortality in India estimates that 45,900 
deaths occur annually in India due to snake bite. The states of Uttar Pradesh, Andhra Pradesh and Bihar share the highest burden of this mortality. Majority of deaths occurred in rural areas, especially during monsoon season and involved young males ${ }^{(2)}$.

More than 2700 species of snakes exist in the world, of which only 450 have front fangs making them capable of injecting venom during bite. The distribution of various types of venomous snakes in different parts of the world is different and therefore, the clinical manifestations and type of anti-venom treatment also differ accordingly. In addition, differences in clinical manifestations of snake bite due to same species in different geographical regions have also been seen and attributed to variations in type of venom ${ }^{(3)}$. India harbors more than 250 species and subspecies of snakes, of which about 50 are venomous ${ }^{(4)}$. In India, "big four" medically important species considered are Naja naja, Bungarus caeruleus, Daboia russelii and Echis carinatus ${ }^{(5)}$.

Local features of snake bite envenomation include pain and swelling. Systemic features include tachycardia, hypotension, pulmonary edema and renal dysfunction. However, renal dysfunction is the most devastating manifestation associated with snake bite, especially in form of Acute Kidney Injury (AKI). In India, AKI is mostly associated with Russell's viper (13\%) and E. carinatus $(32 \%)$. Oliguria or anuria generally develops within a few hours to as late as 96 hours after the snake bite ${ }^{(3)}$. Urine may show gross or microscopic hematuria, hemoglobinuria and proteinuria (less than $500 \mathrm{mg} / 24 \mathrm{~h})^{(6)}$.

The current study was undertaken to evaluate clinical profile of snake bite patients presenting to our institute, to determine the early clinical and biochemical predictors of developing AKI following snake bite and to determine the incidence of AKI.

\section{Material and Methods}

The present prospective observational study was conducted in a minimum of 200 snake bite patients, in department of General Medicine of tertiary care hospital. Ethical approval was taken for the study from institutional ethical committee and written informed consent was obtained. Nondiabetic, non-hypertensive, non-pregnant snake bite patients (aged > 12 years) with history of probable snake bite, symptoms and signs of mild / moderate/severe envenomation including neurological involvement, were included in the study. Snake bite was confirmed from their history and signs, presence of bite marks (fang marks) at the alleged site, identification of snake if possible (dead or alive-if bought by patient or relatives).

Exclusion criteria included - Patients diagnosed with hypertension or diabetes or patients having past history of active renal disease or pre-existing renal disease (clinical and/or laboratory evidence of chronic kidney disease - baseline serum creatinine $>1.5 \mathrm{mg} / \mathrm{dl}$ prior to snake-bite or ultrasonography is suggestive of bilateral small kidneys or loss of cortico-medullary differentiation or obstructive nephropathy or any other significant renal pathology), patients with previous hepato-billiary disease, patients on chronic non-steroidal anti-inflammatory drugs (NSAID) therapy or nephrotoxic drug therapy. Also, patients having no symptoms or no signs of envenomation or no neurological involvement were also excluded.

A detailed history and clinical examination was done and recorded using predesigned and pretested Performa. Snake bite patients were classified as mild or moderate or severe envenomation $^{(7)}$.

Mild: Local findings only (e.g., pain, local ecchymosis, non-progressive swelling).

Moderate: Progressive swelling, systemic features and/or laboratory abnormalities.

Severe: Respiratory distress, neurologic dysfunction, and/or cardiovascular instability.

Patients were administered tetanus toxoid injection, if not received previously. All patients were given polyvalent Anti-Snake Venom (ASV), administered in a dose of 5 vials $(50 \mathrm{ml}$ or 50 units) in the mild envenomation cases, in a dose of 8 vials ( $80 \mathrm{ml}$ or 80 units) in the moderate cases and in a dose of 10 vials $(100 \mathrm{ml}$ or 100 units $)$ in 
severe envenomation cases, as an intravenous infusion in a drip for over 60 minutes. Repeat ASV dose was given depending on $1^{\text {st }} 20 \mathrm{~min}$ Whole Blood Clotting Time (WBCT) test done after 6 hours after the $1^{\text {st }}$ ASV dose and subsequent ASV dosage was administered depending on subsequent $20 \mathrm{~min}$ WBCT test 6 hourly till two consecutive 20min WBCT tests were normal ${ }^{(8)}$. Neuro-paralytic cases were administered 10 vials (100 ml or 100 units) as first dose. If no response after 1 hour, repeat dose of 10 vials (100 ml or 100 units) were administered. Further, if no response after 2 hours, then, patient was treated and observed for neuro-paralysis recovery, without any further ASV dose. Antibiotics, hydration, diuretics, vaso-pressor support, blood/blood products, renal replacement therapy was given to the indicated patients.

The patients were followed up from admission for upto 3 days for development of AKI which was defined using urine output, serum creatinine or Glomerular Filtration Rate (GFR). GFR was calculated using Cockcroft-Gault formula ${ }^{(9)}$. Clinical observation and biochemical parameters were looked for and recorded using predesigned and pretested Performa during three days of follow up after the snakebite.

\section{Clinical Parameters}

Included were Fever, Heart rate, Blood pressure (Hypotension), Site of bite, Local tenderness, Local swelling, Progression of swelling, Oozing from wound, Bleeding from wound, Wound complications (blister, gangrene and compartment syndrome), Bleeding manifestations (petechiae, purpura, ecchymosis and conjunctival hemorrhages), Regional lymphadenopathy and Neurological signs.

\section{Biochemical Parameters}

Included were Urine output (in $\mathrm{ml}$ ), Colour of urine, Urine analysis (Red Blood Cells $\{\mathrm{RBC}\}$, Hemoglobinuria, and Proteinuria), Blood Urea, $\mathrm{Sr}$ Creatinine, GFR, Total Leukocyte Count $\{$ TLC $\}$,
Platelet count, International Normalized Ratio \{INR \}, Bilirubin-Indirect, Sr. Potassium.

Snake-Bite Time and ASV Related Parameters: Included were Bite-to-initial ASV administration time, 20min WBCT Prolongation time and Total dose of ASV.

For comparison, patients were classified into two groups based upon presence or absence of AKI (AKI and No-AKI). Further follow-up of the patient was done for 3 days.

Statistical Analysis

Continuous variables in the two groups were expressed as mean \pm standard deviation. For comparison of categorical variables, Pearson's Chi-square test was used. Fischer exact test was used for small numbers. For continuously distributed variables, Student's ' $t$ ' test for the significance of difference between the means of two independent samples was used. $\mathrm{P}$ value of 0.05 or less was considered to be significant.

Observations and results

Out of 200 snake bite patients included in present study, $63(31.5 \%)$ patients developed AKI.

Table 1 show comparison of mean age, sex (male/female), residence (rural/urban) and bite to initial ASV time with development of AKI in snake bite patients. The average bite-to-initial ASV administration time in AKI group was higher (11.46 \pm 14.03$)$ compared to NON-AKI group $(6.28 \pm 8.11)$, which was highly significant.

The presenting symptoms were local pain (87\%), nausea /vomiting (66\%), bleeding manifestations (29\%), fever (6\%), hypotension $(6.5 \%)$ and neurological symptoms (16\%). Table 2 shows association of presenting complaints with development of AKI. $75 \%$ of patients presented with hypotension and $75.86 \%$ (44/58) patients who had bleeding manifestations at the time of presentation developed AKI and the difference was highly significant $(\mathrm{p}<0.001)$. Thus, significant association was found only between hypotension and bleeding manifestation as presenting complaints with development of AKI. One noteworthy observation is that $15.87 \%$ patients with neurological symptoms as presenting 
complaints developed AKI. However, the association was not statistically significant.

Table 3 shows correlation of clinical signs developed in patients with AKI. Out of 23 patients with oozing, 16 (69\%) patients developed AKI. AKI also developed in $10(83 \%)$ patients presented with blisters. AKI developed in all the 4 patients presented with abscess, $7(87.5 \%)$ patients presented with gangrene, $13(92.8 \%)$ patients presented with skin bleeding, 54 (40.6\%) patients presented with cellulitis and 7 patients presented with compartment syndrome developed AKI. Thus, most of the patients who developed wound complications following snake bite (blisters, oozing, abscess, cellulitis, gangrene and comparetment syndrome) ended up in developing AKI.

Table 4 shows $31(62 \%)$ patients who had hemoglobinuria, $28(58.33 \%)$ patients who had albuminuria and 42 (62.69\%) patients who had $\mathrm{RBC}$ in urine, developed AKI.

Table 5 shows decreased mean platelet count in AKI group (1.67 lac $/ \mathrm{mm}^{3}$ ) as compared to NonAKI group ( $\mathrm{p}$ value $<0.0001$ ). INR mean value was increased in AKI group (1.54) as compared to
Non-AKI group (1.13), (p value <0.0001). Indirect bilirubin mean levels was raised in AKI group $(0.91 \mathrm{mg} / \mathrm{dl})$ as compared to Non-AKI group $(0.74 \mathrm{mg} / \mathrm{dl})$ with a significant statistical difference $(p$ value $=0.0044)$. Thus, decreased platelet, raised INR, raised indirect bilirubin levels were found to significantly associate with development of AKI in snake bite patients.

In present study, it was observed that the severity of envenomation increases, risk of developing AKI also increases. The mean ASV received was higher in AKI group (97.3) as compared to NONAKI group (85.43), but the difference was not statistically significant ( $\mathrm{p}$ value $=0.8046$, CI 95\%). 20 min WBCT test was found to be more than 20 minutes in AKI group of patients $(55.5 \%)$ as compared to NON-AKI group (29.18\%) of snakebite patients $\left(\mathrm{chi}^{2}\right.$ value $=12.7926, \mathrm{p}$ value $<0.001$ ). Most of the patients (41) of AKI following snake bite recovered without any renal replacement therapy. Only 22 patients required renal replacement therapy in the form of peritoneal dialysis.

Table 1. Showing comparison of demographic characters with AKI

\begin{tabular}{|l|c|c|c|}
\hline Characteristics & AKI $(\mathrm{n}=63)$ & Non-AKI $(\mathrm{n}=137)$ & P value \\
\hline Age & $39.60 \pm 14.46$ & $44.04 \pm 15.07$ & $0.72,95 \%$ CI \\
\hline Male & $60.31 \%$ & $46.71 \%$ & 0.07 \\
\hline Female & $39.68 \%$ & $53.28 \%$ & 0.07 \\
\hline Rural & $95.24 \%$ & $96.35 \%$ & 0.71 \\
\hline Urban & $4.76 \%$ & $3.65 \%$ & 0.71 \\
\hline Bite to ASV time & $11.46 \pm 14.03$ & $6.28 \pm 8.11$ & $<0.001$, HS 95\% CI \\
\hline
\end{tabular}

Table 2. Showing association of presenting symptoms with AKI

\begin{tabular}{|l|c|c|c|}
\hline Presenting Symptom & AKI $(\mathrm{n}=63)$ & NON-AKI $(\mathrm{n}=137)$ & P value \\
\hline Local pain & 57 & 117 & 0.32 \\
\hline Nausea / vomiting & 46 & 86 & 0.16 \\
\hline Bleeding & 44 & 14 & $<0.001$ \\
\hline Fever & 6 & 7 & 0.24 \\
\hline Hypotension & 9 & 3 & $<0.001$ \\
\hline Neurological & 10 & 22 & 0.97 \\
\hline
\end{tabular}


Table 3. Showing comparison of clinical signs in AKI and NON-AKI group

\begin{tabular}{|l|c|c|c|}
\hline Signs & AKI & NON-AKI & P value \\
\hline Tenderness & 48 & 99 & 0.56 \\
\hline Progressive swelling & 49 & 88 & 0.06 \\
\hline Oozing & 16 & 5 & $<0.001$ \\
\hline Blisters & 10 & 2 & $<0.001$ \\
\hline Abscess & 4 & 0 & 0.003 \\
\hline Cellulitis & 54 & 79 & $<0.001$ \\
\hline Gangrene & 7 & 1 & $<0.001$ \\
\hline Skin bleeding & 13 & 1 & $<0.001$ \\
\hline Compartment syndrome & 7 & 0 & $<0.001$ \\
\hline Regional lymphadenopathy & 32 & 59 & 0.31 \\
\hline
\end{tabular}

Table 4. Comparison of biochemical parameters in AKI and Non-AKI Group

\begin{tabular}{|c|c|c|c|c|c|}
\hline Signs & $\begin{array}{l}\text { Total } \\
\text { cases }\end{array}$ & AKI & $\begin{array}{c}\text { NON- } \\
\text { AKI }\end{array}$ & $\begin{array}{c}\text { CHI square } \\
\text { value }\end{array}$ & $\mathrm{P}$ value \\
\hline Hemoglobinuria present & 50 & 31 & 19 & \multirow{2}{*}{28.7414} & \multirow{2}{*}{$<0.001$} \\
\hline Hemoglobinuria absent & 150 & 32 & 118 & & \\
\hline Albuminuria present & 48 & 28 & 20 & \multirow{2}{*}{21.0754} & \multirow{2}{*}{$<0.001$} \\
\hline Albuminuria absent & 152 & 35 & 117 & & \\
\hline $\mathrm{RBC}$ in Urine present & 67 & 42 & 25 & \multirow{2}{*}{45.4137} & \multirow{2}{*}{$<0.001$} \\
\hline $\mathrm{RBC}$ in Urine absent & 133 & 21 & 112 & & \\
\hline $20 \mathrm{~min} \mathrm{WBCT}$ test $>20 \mathrm{~min}$ & 75 & 35 & 40 & \multirow{2}{*}{12.7926} & \multirow{2}{*}{$<0.001$} \\
\hline $20 \mathrm{~min}$ WBCT test $<20 \mathrm{~min}$ & 125 & 28 & 97 & & \\
\hline
\end{tabular}

Table 5. Comparison of other hematological parameters in AKI and Non-AKI Group

\begin{tabular}{|l|c|c|c|}
\hline Parameter & AKI & Non-AKI & p-value \\
\hline Total Leucocyte Count & $13342.06 \pm 5093.71$ & $12714.31 \pm 5130.74$ & $0.4215, \mathrm{NS}$ \\
\hline Platelet count & $1.67 \pm 0.79$ & $2.61 \pm 1.00$ & $<0.0001, \mathrm{HS}$ \\
\hline INR & $1.54 \pm 0.54$ & $1.13 \pm 0.28$ & $<0.0001, \mathrm{HS}$ \\
\hline Serum Potassium & $3.97 \pm 0.51$ & $3.99 \pm 0.48$ & $0.8205, \mathrm{NS}$ \\
\hline Indirect Bilirubin & $0.91 \pm 0.59$ & $0.74 \pm 0.23$ & $0.0044, \mathrm{HS}$ \\
\hline
\end{tabular}

\section{Discussion}

In India, the incidence of AKI following $\mathrm{E}$. carinatus or Russell's viper bite is $13-32 \%{ }^{(10,11)}$. In the present study, $31.5 \%$ (63 patients) snake bite victims developed AKI. Harshavardhan L et al ${ }^{(12)}$ noted that AKI developed in $14.6 \%$ cases of snake bite, whereas Paul $\mathbf{J}$ et al ${ }^{(13)}$ observed that $43.27 \%$ cases of viper bite were complicated by AKI. Also, Dharod MV et al ${ }^{(14)}$ noted that AKI developed in $30.9 \%$ cases of snake bite. The main cause of this high incidence of AKI is that the people try out all kinds of "bizarre remedies" initially, instead of going to the nearest hospital ${ }^{(12)}$. In present study, high incidence may be due to treatment delay of snake bite patients either due to transport problem or trying some local non- medical treatment before admission in hospital. Further the use of creatinine clearance as diagnostic criteria for AKI allowed a higher precision in AKI diagnosis ${ }^{(13)}$.

However, no significant association was found between age, sex, residence, literacy with development of AKI. In present study, $88 \%$ of the study populations have educational status below $10^{\text {th }}$ standard. However, Harshavardhan L et al ${ }^{(12)}$ found that illiteracy was more among the patients who developed AKI (75\%) due to snakebite. Suchithra Net al ${ }^{(15)}$ found fewer incidences of complications after snakebite envenomation in Kerala where literacy rate is high, as compared to other studies done in India. This relation observed between literacy and AKI may be due to the fact 
that a high literacy status prefers less field work and favors early preference for proper medical treatment.

In present study, the average bite-to-initial ASV administration time in AKI group was higher as compared to NON-AKI group, which was highly significant and was reported in various studies ${ }^{(13-}$ 15). Thus, increased bite-to-initial ASV administration time can be considered as an early predictor of AKI in snakebite patients suggesting that a shorter bite-to-initial ASV administration time can help in preventing AKI in snake bite patients.

The presenting symptoms in present study groups were local pain, nausea /vomiting, bleeding manifestations, fever, hypotension and neurological symptoms (double vision, dyspnea and dysphagia). 9 (14.28\%) patients in AKI group presented with hypotension while $54(85.72 \%)$ patient did not have hypotension. In NON-AKI group, only 3 patients (2.19\%) had hypotension while $134(97.81 \%)$ patients did not have hypotension. The difference was statistically significant ( $\mathrm{p}$ value $<0.001$ ). Also, in AKI group, $44(69.84 \%)$ patients had bleeding manifestation while only $14(10.22 \%)$ of patients in NON-AKI group had bleeding manifestation with a statistically significant difference ( $p$ value $<0.001$ ). The presence of bleeding manifestations was identified as independent predictors of poor outcome in snake bite patients (16). Thus, hypotension and bleeding manifestations can be considered as predictors of AKI in snake bite patients. Findings in present study correlated with various studies ${ }^{(14-16)}$. A notable observation in present study was that, $15.87 \%$ patients with neurological symptoms as presenting complaints developed AKI. However, this association was not statistically significant.

Snake bite patients with envenomation features were included in three categories: mild envenomation, moderate envenomation and severe envenomation. In present study, it was found that AKI was more evident in moderate envenomation and severe envenomation cases. $35.65 \%$ cases with moderate envenomation and $45.45 \%$ cases with severe envenomation developed AKI. Thus, as the severity of snake bite increases, the risk of developing AKI increases and the trend was statistically significant ( $p$ value $<0.001$ ). A number of factors contribute to it, like bleeding, hypotension, circulatory collapse, intravascular hemolysis, disseminated intravascular coagulation, microangiopathic hemolytic anemia and the direct nephrotoxicity of venom ${ }^{(17)}$. Similar observations were made by Dharod MV et al ${ }^{(14)}$ and Athappan $\mathrm{G}$ et $\mathrm{al}^{(16)}$ in their respective studies.

Wound complication usually correlate with amount of venom released after snake bite ${ }^{(5)}$. However, severe envenomation without much swelling or local complications can occur if the venom directly enters the blood stream ${ }^{(18)}$. Other causes for wound complication include poor wound hygiene (applying substances over wound, cutting the wound for letting out venom) and tight tourniquet application ${ }^{(5)}$. In present study, most of the patients who developed wound complications (like blisters, oozing, abscess, cellulitis, gangrene and compartment syndrome) following snake bite, ended up developing AKI. A significant association was found between each of these wound complication and development of AKI. Thus, development of wound complications can be considered as an indicator for development of AKI in snake bite patients. This was in agreement with the study of Harshavardhan Let al ${ }^{(12)}$ and Athappan Get al ${ }^{(16)}$.

The presence of lab parameters (Urinary) like hemoglobinuria, albuminuria and $\mathrm{RBC}$ in urine was observed in present study. A higher incidence of these parameters was noticed in AKI group when compared to NON-AKI group with a significant correlation. Hence, presence of hemoglobinuria, albuminuria and RBC in urine can be considered as an indicator for development of AKI in snake bite patients. These results were comparable with various studies ${ }^{(14,16,18)}$.

The lab parameters (hematological) analyzed in snake bite patients in present study were total leukocyte count, platelet count, INR, indirect bilirubin and serum potassium. Low platelet, raised INR and raised indirect bilirubin were found to be significantly associated with 
development of AKI in snake bite patients and can be considered as predictors for development of AKI in snake bite patients. These findings correlated with different studies ${ }^{(15,14,19)}$.

ASV was received by snake bite patients based on envenomation features (mild, moderate and severe). Bleeding manifestations of coagulopathy in the present study was demonstrated as prolonged 20min WBCT test (> $20 \mathrm{~min}$ ). $20 \mathrm{~min}$ WBCT test was used to screen for hemotoxic effects of snake bite and subsequently, 6 hourly 20 min WBCT test was used to assess whether effective dose of ASV was received by snake bite patients. 20min WBCT test was found to be more than 20 minutes in AKI group of patients $(55.5 \%)$ as compared to NON-AKI group (29.18\%) of snake bite patients and the difference was significant. Intravascular coagulation and DIC are an important factor that increases the risk of AKI, positive 20min WBCT test can also be used as screening tool for development of AKI in snake bite patients. The results were comparable with the study of Paul J et al ${ }^{(13)}$ and Athappan $G$ et al (16)

The Indian national snake bite protocol ${ }^{(8)}$ and previous editions of World Health Organization (WHO) guidelines of 2010 (20) recommend immediate administration of 5 vials. Recent WHO guidelines of $2016^{(5)}$ recommends 4-6 vials in the case of Echis carinatus; and 10 vials for patients envenomed by the other species, with repeated dosing if the patient fails to improve, to a maximum of 20 vials. In present study, the mean ASV received was found to be higher in AKI group (94.4units, nearly 95ml) as compared to NON-AKI group (78.9units, nearly $79 \mathrm{ml}$ ) but the difference was not statistically significant. This result correlated with previous studies ${ }^{(13,16)}$.

In present study, most of the patients of AKI following snake bite recovered without any renal replacement therapy and recovered on conserveative management. Only 22 patients required renal replacement therapy in the form of peritoneal dialysis which was readily available in the hospital.

\section{Limitations of the study}

The offending snake species could not be identified. Also, ELISA test to identify snake venom was not available due to technical constraints. Since the patients were followed up for only 3 days, long term outcome and late complications were not identified. The hospital setting in which study were conducted did not had emergency hemodialysis facility, so a comparison between peritoneal dialysis and hemodialysis on outcome of AKI following snake bite, was not possible.

\section{Conclusion}

We found that early clinical predictors for the development of AKI in snake bite patients are bleeding manifestations, hypotension, tenderness, progressive swelling, wound complications (blisters, oozing, abscess, cellulitis, gangrene and compartment syndrome) as well as prolonged bite-to-initial ASV administration time. Whereas early biochemical predictors for the development of AKI in snake bite patients are hemoglobinuria, albuminuria, RBC in urine, elevated INR, elevated indirect bilirubin, decreased platelets and prolonged 20min WBCT test.

More research is needed to scientifically validate the above findings, especially to find correlation of these clinical and biochemical predictors with the development of AKI so that early identification of prognostic risk factors and proper timely management may reduce patient's morbidity and mortality.

\section{Acknowledgement}

The authors would like to thank the department of General Medicine and administration of Government Medical College, Nagpur, Maharashtra, for permission to study and providing facility to carry out the work.

\section{References}

1. Kasturiratne A, Wickremasinghe AR, de Silva N, Gunawardena NK, Pathmeswaran A, Premaratna Ret al. The Global Burden of Snakebite: A Literature Analysis and 
Modelling Based on Regional Estimates of Envenoming and Deaths. PLoS Med 2008;5:218.

2. Mohapatra B, Warrell DA, Suraweera W, Bhatia P, Dhingra N, Jotkar RM, et al. Snakebite mortality in India: A nationally representative mortality survey. PLo S Negl Trop Dis 2011;5:1018.

3. Chugh KS. Snake-bite-induced acute renal failure in India. Kidney international 1989;35(3):891-907.

4. Williams D, Gutiérrez JM, Harrison R, Warrell DA, White J, MD, Winkel KD, Gopalakrishnakone P. The Global Snake Bite Initiative: an antidote for snake bite, WHO 2007.

5. WHO. Regional Office for South-East Asia. Guidelines for the management of snake-bites 2016, 2nd edition.

6. Sitprija V. Snakebite nephropathy (Review Article). Nephrology 2006;11(5):442-448.

7. Aurebach PS, Norris RL. Disorders caused by venoumous snake bites and marine exposures. Harrisons principle of internal medicine, $18^{\text {th }}$ edition 2012; vol 2 (396):3566-3568.

8. Directorate General of Health Services, Ministry of Health and Family Welfare Government of India. National Snakebite ManagementProtocol2009.

9. Cockcroft DW, Gault MH. Prediction of creatinine clearance from serum creatinine. Nephron 1976;16(1):31-41.

10. Monteiro FN, Kanchan T, Bhagavath P, Kumar GP, Menezes RG, Yoganarasimha K. Clinico-epidemiological features of viper bite envenomation: A study from Manipal, South India. Singapore Med J 2012;53:203-207.

11. Lavonas EJ, Tomaszewski CA, Ford MD, Rouse AM, Kerns WP., 2nd Severe puff adder (Bitis arietans) envenomation with coagulopathy. J Toxicol Clin Toxicol 2002;40:911-918.
12. Harshavardhan L, Lokesh AJ, Tejeshwari HL, Halesha BR, Siddharama S. A Study on the Acute Kidney Injury in Snake Bite Victims in A Tertiary Care Centre. Journal of Clinical and Diagnostic Research 2013;5:853-856.

13. Paul J, Dasgupta S. Early prediction of acute kidney injury by clinical features of snakebite patients at the time of hospital admission. North American journal of medical sciences 2012;4(5):216.

14. Dharod MV, Patil TB, Deshpande AS, Gulhane RV, Patil MB, Bansod YV. Clinical Predictors of Acute Kidney Injury Following Snake Bite Envenomation. N Am J Med Sci 2013;5(10):594-599.

15. Suchithra N, Pappachan JM, Sujathan P. Snakebite envenoming in Kerala, south India: Clinical profile and factors involved in adverse outcomes. Emerg Med J 2008;25:200-204.

16. Athappan G, Balaji MV, Navaneethan U, Thirumalikolundusubramanian P. Acute Renal Failure in Snake Envenomation: A Large Prospective Study. Saudi J Kidney Dis Transpl 2008;19(3):404-410.

17. KohliH S, Sakhuja V. Snake Bites and Acute Renal Failure. Saudi J Kidney Dis Transplant 2003;14(2):165-176.

18. Ali G, Kak M, Kumar M, Bali SK, Tak SI, Hassan G, Wadhwa MB. Acute renal failure following echiscarinatus (sawscaled viper) envenomation. Indian $\mathbf{J}$ Nephrol 2004;14:177-181.

19. Vijeth SR, Dutta TK, Shahapurkar J. Correlation of renal status with hematologic profile in viperine bite. Am. J. Trop. Med. Hyg 1997;56:168-176.

20. Warrell DA. Guidelines for the management of snake-bites. Guidelines for the management of snake-bites. 2010. 